Special issue in honor of Prof. George C. Papageorgiou

\title{
Exposed red leaves display adaptive adjustments in chlorophyll and photosystem ratios compatible with the shade imposed by anthocyanin accumulation
}

\author{
K. ZELIOU, A. KYZERIDOU, and Y. PETROPOULOU ${ }^{+}$ \\ Laboratory of Plant Physiology, Department of Biology, University of Patras, Patras GR-26504, Greece
}

\begin{abstract}
Foliar anthocyanins shape a peculiar shade in a red leaf's interior leading to uneven energy distribution between the two photosystems. Accordingly, a readjustment of PSII/PSI stoichiometry could restore excitation balance. To test this hypothesis, $77 \mathrm{~K}$ fluorescence emission spectra of thylakoids from green and red leaves of seven species with different pigment profiles were compared. The ratio of $\mathrm{F}_{686} / \mathrm{F}_{736}$ served as an indication of the PSII/PSI functional ratio. To avoid possible species-dependent differences in the measured parameters, plants showing intra-individual, intra-species, or intra-leaf variation in the expression of the anthocyanic character were used. Red leaves or red leaf areas displayed higher PSII/PSI ratio, irrespectively of species and anthocyanin accumulation pattern. PSII/PSI ratio declined in parallel with anthocyanin decrease. In five species, red leaves displayed also a lower Chl $a / b$ ratio. We conclude that red leaves growing in full sunlight develop adaptive adjustments in their chlorophyll and photosystem ratios, compatible with the shade-acclimation syndrome.
\end{abstract}

Keywords: fluorescence emission spectra; foliar anthocyanins; photosystem ratio; shade-acclimation syndrome.

\section{Introduction}

Some plants accumulate anthocyanins in their leaves at levels high enough to mask the green chlorophyll (Chl) color. The major leaf anthocyanin is cyanidin-3-glucoside (Harborne 1976), absorbing strongly in the green/yellow, lesser in the blue, and negligibly in the red part of the spectrum (Neill and Gould 2000, Hughes and Smith 2007, Gould et al. 2018, Landi et al. 2021). Accordingly, anthocyanic leaves appear red to dark brown, depending on the contents of co-existing chlorophylls. Foliar anthocyanins may be found in epidermises (upper and/ or lower), mesophyll (palisade and/or spongy cells), trichomes, and they reside exclusively in the vacuole (Hrazdina et al. 1978, Lee and Collins 2001, Kytridis and Manetas 2006). Although in some species the anthocyanic trait is permanent, the transient leaf redness is more common and may be induced by developmental or environmental agents. In the first case, young or senescing leaves appear red while being green at the mature stage. In the latter, green fully expanded leaves turn temporarily red as a response to UV-B radiation, nutrient deficiency, wounding, pathogen attack, or low temperatures accompanied by high light (Gould et al. 2002a, Steyn et al.

\section{Highlights}

- Red leaves display a higher PSII/PSI ratio, compared to their green counterparts

- PSII/PSI ratio declines in parallel with the decrease of anthocyanin content

- Red leaves displayed also a lower $\mathrm{Chl} a / b$ ratio
Received 6 October 2021

Accepted 1 December 2021

Published online 13 January 2022

${ }^{+}$Corresponding author

e-mail: petropo@upatras.gr

Abbreviations: Anths - anthocyanins; Car - total carotenoids; Chl - chlorophyll; FR - far-red light; R - red light. Acknowledgments: This paper is dedicated to the memory of the late Dr. George C. Papageorgiou for his important contribution to photosynthesis research and we are honored to participate in this special issue.

Conflict of interest: The authors declare that they have no conflict of interest. 
2002, Close and Beadle 2003, Gould 2004, Manetas 2006, Hughes 2011).

Since anthocyanins absorb visible radiation, their accumulation in leaves represents a photosynthetic cost because of the competition with Chls and carotenoids (Car) for photon capture (Merzlyak et al. 2008). Assuming that this cost should be counterbalanced by some advantages of leaf redness, numerous hypotheses for an adaptive significance of the anthocyanin accumulation have been proposed (for reviews see Chalker-Scott 1999, Hoch et al. 2001, Steyn et al. 2002, Close and Beadle 2003, Gould 2004, Lev-Yadun et al. 2004, Manetas 2006, Archetti et al. 2009, Hughes 2011). Regardless of the ascribed function(s), however, anthocyanins modify quantitatively as well as qualitatively the internal light environment of a leaf. Pietrini and Massacci (1998), using an indirect estimation, calculated that the chloroplasts of an anthocyanic leaf may enjoy as much as $40 \%$ lower photosynthetically active radiation than its anthocyaninless counterpart. In addition, it has been repeatedly shown that red leaves display an array of morphological and physiological characteristics, partly compatible with the shade acclimation syndrome (Gould et al. 2002a, Manetas et al. 2003, Hughes and Smith 2007, Hughes et al. 2007, Kyparissis et al. 2007, Kytridis et al. 2008, Zeliou et al. 2009).

It is well established in the literature that almost all photosynthetic parameters of leaves and chloroplasts, especially pigment contents and their ratios, are strongly affected by both the quantity and quality of light they receive. Compared to the exposed sites, under a dense plant or forest canopy prevails diffuse light of low intensity, greatly depleted in red and blue while enriched in green and far-red photons. Thus, shade leaves possess significantly lower Chl $a / b$ values than the corresponding sun leaves, since they invest primarily in higher amounts of light-harvesting chlorophyll proteins and lower number of reaction center pigment proteins (Anderson et al. 1995, Lichtenthaler and Babani 2004, Lichtenthaler et al. 2007, 2013). The internal light microenvironment shaped by an anthocyanin screen, however, is not neutral or canopy-like since, in contrast to natural shade, the green/yellow part of the penetrated light is strongly affected (Karabourniotis et al. 1999, Gould et al. 2002b, 2018). Hence, in the interior of an anthocyanic leaf, the light is FR-enriched (as in natural shade) but green/yellow depleted. This spectral selectivity may be superimposed on the already lower internal photon fluence rates and further modify a red leaf's photosynthetic physiology. According to Kyparissis et al. (2007), this hypothesis could explain some deviations from the classical shade acclimation syndrome observed in the permanently red leaves of Prunus cerasifera.

Based on the above, we argued that the selective attenuation mainly of green/yellow and, to a lesser degree, blue light by anthocyanins represents a potential loss for PSII, which preferentially absorbs these spectral bands (Glick et al. 1985, Pfannschmidt 2005, Hogewoning et al. 2012). Accordingly, an adjustment in photosystem stoichiometry could be inferred (Chow et al. 1990), to compensate for the uneven spectral distribution of photons within a red leaf. Towards that aim, $77 \mathrm{~K}$ fluorescence spectra of dilute thylakoid preparations obtained from green and red leaves of various species with different pigment profiles were compared. The corresponding $\mathrm{F}_{686} / \mathrm{F}_{736}$ ratio from each leaf type was used as a relative indication of the PSII/PSI functional analogy (Krause and Weis 1991, Papageorgiou and Govindjee 2004, Lamb et al. 2018). The main criteria for species selection were (1) the avoidance of possible species-dependent differences in the measured parameters and any confounding effect from the different irradiation history of the selected leaves and (2) the examination of species with different profiles of anthocyanin accumulation (tissue localization and/or inductive agent). Thus, we included plants displaying intra-individual, intra-species, or intraleaf variation in the expression of the anthocyanic trait, accumulating anthocyanins permanently or transiently in the epidermis(es) or the mesophyll.

\section{Materials and methods}

Plant material and sampling: To meet the requisites mentioned in the 'Introduction', the following seven species with transient or permanent anthocyanin accumulation in their young or mature leaves were used as experimental material:

Ricinus communis L.: perennial shrub cultivated as ornamental in the Patras University Campus. During spring and autumn produces red young leaves, which become gradually green upon maturation. Two individuals were used.

Photinia $\times$ fraseri Dress.: perennial shrub with young red and mature green leaves. As with $R$. communis, new leaf production occurs during spring and autumn under Mediterranean conditions. Eight individuals were purchased from a local nursery and cultivated in plastic pots in a sun-lit site of Patras University Campus.

Rosa sp.: two varieties possessing red or green young leaves were selected, while the mature leaves of both are green. New leaves burst during spring. Six plants (three from each variety) were purchased from a local nursery and cultivated in plastic pots in a sun-lit site of Patras University Campus.

Quercus coccifera L.: Mediterranean evergreen sclerophyll shrub, growing in wild in the neighborhood of Patras University Campus. This species displays intra-species variation in the expression of leaf redness, i.e., some individuals possess dark red young leaves while other neighboring plants (occupying the same habitat) possess green young ones (Manetas et al. 2003). New leaves burst during the spring (early April to mid-May) at the top of older branches. The mature leaves of both phenotypes are green. From previous observations at the same field site, it was confirmed that the same plants produce red or green young leaves every year (i.e., there is stability in the expression of leaf redness among individuals). Thus, 12 tagged individuals ( 6 per phenotype) were used.

Cistus creticus L.: evergreen malacophyllous Mediterranean shrub growing also in wild in the vicinity of Patras University Campus. Certain individuals of this 
species turn red at mid-winter and resume their green color in late spring (after the shedding of old leaves) while neighboring individuals under the same environmental conditions remain invariably green (Kytridis and Manetas 2006, Kytridis et al. 2008, Zeliou et al. 2009). As with $Q$. coccifera, there is a constancy in the expression of the anthocyanic trait among individuals. Throughout the remaining seasons (from late spring to mid-winter), both phenotypes have green leaves. New leaves (green) burst in mid-April. Ten tagged plants (five per phenotype) were used.

Prunus cerasifera Ehrh. (var. atropurpurea) grafted at the base of Prunus domestica L. rootstock: the two species form small two-stemmed trees, with a permanently green (P. domestica) and a permanently red-leafed ( $P$. cerasifera) stem. Both stems are winter deciduous. Two plants grown as ornamentals in the Patras University Campus were used.

Coleus blumei Benth, a tropical understory variegated species, possessing chlorophyllous anthocyanin-rich ('red areas') and anthocyanin-less ('green areas') spots in the same leaf. Twelve individuals of about the same age cultivated in plastic pots in a shaded site of Patras University Campus were used.

All plants, growing side by side under the same environmental conditions, were equally exposed to direct solar radiation except $C$. blumei, which received $\sim 200 \mu \mathrm{mol}$ (photon) $\mathrm{m}^{-2} \mathrm{~s}^{-1}$ (maximum mid-day PAR intensity on plant apex). During the whole experimental period, the wild-growing plants received only natural precipitation while the cultivated plants were well watered.

Sampling was performed always on clear days and each measuring date south-facing (i.e., fully exposed) green and red leaves from each species were compared. In $R$. communis and $P$. × fraseri, young and mature leaves co-occur on the same individual, because of the extended duration of leaf development. Thus, two age classes of young leaves (referred to as red A and red B, respectively) were compared with mature green. Leaf selection was based on their color and position to the branch, numbered from the apex. In the case of $C$. creticus, measurements were performed in mature leaves of the two phenotypes at frequent intervals, during both the 'green' (i.e., when the leaves of both phenotypes are green) and the 'red' (i.e., when leaves of some individuals turn red) period of the year. Sampling started before the appearance of redness (in early December) and ended in late spring when the red phenotype reverted to green.

On each measuring date, an adequate number of green and/or red leaves from each individual of a given species were harvested early in the morning, put in air-tight plastic bags with moistened filter paper, and transferred immediately to the laboratory for further experimentation. Discs (2-4) of known diameter were punched from each leaf to be used for pigment extraction. The remaining tissue of red or green leaves (without the main vein) was pooled, cut in small segments, and put in a mortar with a small volume of liquid $\mathrm{N}_{2}$ to proceed for thylakoid isolation. In the case of $C$. blumei, an adequate number of discs from the corresponding 'red' and 'green' areas of each mature leaf was cut and pooled separately.

The location of anthocyanins was assessed by microscopic examination of free-hand leaf cross-sections from each species in conjunction with previous observations (Burger and Edwards 1996, Manetas et al. 2003, Kytridis and Manetas 2006, Kyparissis et al. 2007). Anthocyanin localization and the comparisons performed within each species are presented in the text table below.

Thylakoid isolation: All the procedures took place at $4^{\circ} \mathrm{C}$. Thylakoids were prepared using the method of Šiffel et al. (2000) with some modifications (Yiotis et al. 2009). Briefly, the leaf segments (or discs) were grinded in the 'isolation medium' (0.4 M sorbitol, $5 \mathrm{mM} \mathrm{MgCl}, 10 \mathrm{mM}$ $\mathrm{KCl}, 1 \mathrm{mM} \mathrm{MnCl}_{2}, 1 \mathrm{mM}$ sodium ascorbate, $0.5-1 \%$ bovine serum albumin, $50 \mathrm{mM}$ Tricine/KOH, $\mathrm{pH}$ 7.6). After two sequential centrifugations (at 1,500 $\times g$ for $2 \mathrm{~min}$ and $8,000 \times g$ for $10 \mathrm{~min}$ ) the pellet was resuspended in 'washing medium' $(0.33 \mathrm{M}$ sorbitol, $5 \mathrm{mM}$ $\mathrm{MgCl}_{2}, 10 \mathrm{mM} \mathrm{KCl}, 1 \mathrm{mM} \mathrm{MnCl} 2,0.05 \%$ bovine serum albumin, $50 \mathrm{mM}$ Tricine/KOH, $\mathrm{pH}$ 7.6). Following two consecutive resuspensions-centrifugations at 8,000 $\times g$ for $10 \mathrm{~min}$, the final pellet was resuspended in $2 \mathrm{ml}$ of 'washing medium' and served as the thylakoid preparation. For each fluorescence measurement, a small aliquot (200$400 \mu \mathrm{l})$ of the thylakoid sample was used after appropriate dilution.

\begin{tabular}{|c|c|c|}
\hline Species tested & Anthocyanin location & Leaf comparison \\
\hline \multicolumn{3}{|l|}{ Intra-individual variation } \\
\hline $\begin{array}{l}\text { Ricinus communis } \\
\text { Photinia } \times \text { fraseri }\end{array}$ & $\begin{array}{l}\text { upper epidermis } \\
\text { whole mesophyll }\end{array}$ & young red $v s$. mature green \\
\hline Intra-species variation & & \\
\hline $\begin{array}{l}\text { Rosa sp. } \\
\text { Quercus coccifera }\end{array}$ & $\begin{array}{l}\text { both epidermises } \\
\text { mesophyll layers (subepidermal) }\end{array}$ & young red $v s$. young green \\
\hline $\begin{array}{l}\text { Cistus creticus } \\
\text { Prunus cerasifera } \times \text { Prunus domestica }\end{array}$ & $\begin{array}{l}\text { palisade mesophyll } \\
\text { whole mesophyll }\end{array}$ & mature red $v s$. mature green \\
\hline $\begin{array}{l}\text { Intra-leaf variation } \\
\text { Coleus blumei }\end{array}$ & upper epidermis & red $v s$. green areas of the same leaf \\
\hline
\end{tabular}


Low-temperature fluorescence spectra: $77 \mathrm{~K}$ fluorescence emission spectra of dilute thylakoid preparations were recorded using a spectrofluorimeter $(F-2500$, Hitachi, High Technologies Corporation, Japan), equipped with a liquid-nitrogen sample devise and a red-sensitive photomultiplier, as previously described (Yiotis et al. 2009). The calculated $F_{686} / F_{736}$ ratio served as a relative indication of the PSII/PSI ratio (Krause and Weis 1991). Care was taken to keep the Chl concentration of the probed sample at low contents $\left(2-4 \mu \mathrm{g} \mathrm{ml}{ }^{-1}\right.$, as judged from preliminary trials), to avoid self-absorption of Chl fluorescence (Weis 1985, Lamb et al. 2018). Total Chls of the thylakoid samples eluted in pure methanol were estimated by the equations of Lichtenthaler and Wellburn (1983), using a double-beam spectrophotometer (Shimadzu UV-160A). To prevent condensation, a water vapor-free airstream was supplied in the Dewar cuvette of the spectrofluorimeter. Excitation was set at $490 \mathrm{~nm}$ (slit width $10 \mathrm{~nm}$ ) and emission was recorded between 670-800 nm (slit width $2.5 \mathrm{~nm}$ ). Every thylakoid sample was measured three times. Data were exported in the form of a smoothed curve and datasheet with the Hitachi FL Solution software. Raw fluorescence spectra were smoothed with the Savitsky-Golay method and analyzed by a multi-peak fitting process. Three Gaussian bands at 685,695 , and $735 \mathrm{~nm}$ were used for spectra fitting.

Pigment determination: The discs sampled before thylakoid isolation were frozen in a mortar by adding a small volume of liquid nitrogen and pigments extracted in dim light with pure methanol in the presence of a small amount of $\mathrm{CaCO}_{3}$. The extract was centrifuged at $5,000 \times g$ for $10 \mathrm{~min}$ at $4^{\circ} \mathrm{C}$ and the supernatant was used for pigment determination. Chls were measured spectrophotometrically, using a Shimadzu UV-160A double-beam spectrophotometer (Shimadzu Deutschland $\mathrm{GmbH}$, Duisburg, Germany), and concentration was estimated according to the equations of Lichtenthaler and Wellburn (1983). For anthocyanin determination, the methanolic leaf extracts were acidified with $1 \% \mathrm{HCl}$ and absorbance was scanned from 400-700 $\mathrm{nm}$. The peak anthocyanin absorbance (528-532 nm, depending on species) was corrected for pheophytin absorbance at this wavelength (Lindoo and Caldwell 1978) and transformed to actual concentrations by using the mean molar absorption coefficient according to Murray and Hackett (1991).

Statistics: Significance of differences in the measured parameters between green and red leaves of each species was assessed with Student's t-test (SPSS v. 20.0 statistical package, IBM-SPSS Statistics, Armonk, NY, USA). In the case of $C$. creticus, statistically significant differences between green and red leaves were assessed separately for the 'green' and the 'red' period.

\section{Results}

Fig. 1 (left panels) presents $77 \mathrm{~K}$ fluorescence spectra recorded from dilute thylakoid preparations of red and green leaves of the seven species tested. To facilitate comparison, within each species, the fluorescence values of red leaves were normalized to those of the corresponding green leaf at $\lambda=736 \mathrm{~nm}$. In all cases, the $77 \mathrm{~K}$ spectra showed a typical profile with two main bands peaking at 686 and $736 \mathrm{~nm}$ related to the core antenna of PSII and LHCI-PSI, respectively. The third band at $695 \mathrm{~nm}$, arising from the CP47 PSII core antenna, appears as a shoulder. From the corresponding fluorescence values of each leaf type and species, the $\mathrm{F}_{686} / \mathrm{F}_{736}$ ratio (indicative of the relative functional analogy of the two photosystems, PSII/ PSI) was calculated (Fig. 1, right panels).

As it is shown, in all species red leaves displayed higher PSII fluorescence and a higher $\mathrm{F}_{686} / \mathrm{F}_{736}$ ratio compared to their green counterparts, irrespectively of their anthocyanin accumulation pattern. More specifically, in $R$. communis and $P . \times$ fraseri (Fig. $1 A, B$ ), where leaves of the different developmental stages were compared, PSII/PSI ratio declined in parallel to the gradual loss of anthocyanins. A higher $\mathrm{F}_{686} / \mathrm{F}_{736}$ ratio of red leaves was also confirmed with the next four species (Fig. $1 C-F$ ), where leaf morphs of about the same physiological age, either young (Rosa sp., Q. coccifera) or mature (C. creticus, $P$. cerasifera $\times P$. domestica), were compared. The same applies for C. blumei (Fig. $1 G$ ), where the comparisons concern adjacent red and green chlorophyllous spots of the same mature leaf. In the case of $C$. creticus (Fig. $1 E$ ), the emission spectra corresponded to thylakoids isolated from the two phenotypes during the 'red' period of the year. During the 'green' period (when the leaves of both phenotypes are green), there was no difference in fluorescence emission.

Area-based anthocyanin and total $\mathrm{Chl}$ concentrations along with $\mathrm{Chl} a / b$ ratios of red and green leaves from all species tested are given in Fig. 2. As expected from their colors, in most of the cases, green leaves possess negligible (less than $2 \%$ ) or very low (5-9\% in C. creticus and $C$. blumei, respectively) anthocyanin concentrations compared to the corresponding reds. The first two species displayed a common pigment profile, i.e., the gradual loss of anthocyanins upon maturation was accompanied by an increase in Chl concentration (as expected), without any difference in the $\mathrm{Chl} a / b$ ratio. Thus, in $R$. communis, red leaves had $\mathrm{ca} 45 \%(\mathrm{red} \mathrm{A})$ and $64 \%(\operatorname{red~B})$ of the $\mathrm{Chl}$ content of the mature green ones, while their anthocyanins were $\sim 120$ - and 85 -fold higher, respectively (Fig. $2 A$ ). In $P$. $\times$ fraseri, a delayed-greening species, total Chls increased more sharply with age, with young leaves containing $18 \%$ (red A) and 35\% (red B) of the corresponding mature ones. Concerning anthocyanins, though they didn't differ between the two young age classes, they were $\sim 55$-fold higher in red compared to green leaves (Fig. 2B).

In the remainder species, compared to green, red leaves displayed always a slightly, yet significantly, lower (7$17 \%$, depending on species) $\mathrm{Chl} a / b$ ratio. Concerning total Chls, no difference was observed in three species (Rosa sp., Q. coccifera, C. blumei; Fig. 2C,D,G, respectively) while in $C$. creticus and $P$. cerasifera $\times P$. domestica, red leaves had $\sim 30 \%$ lower Chl values (Fig. $2 E, F$ ). We have to 

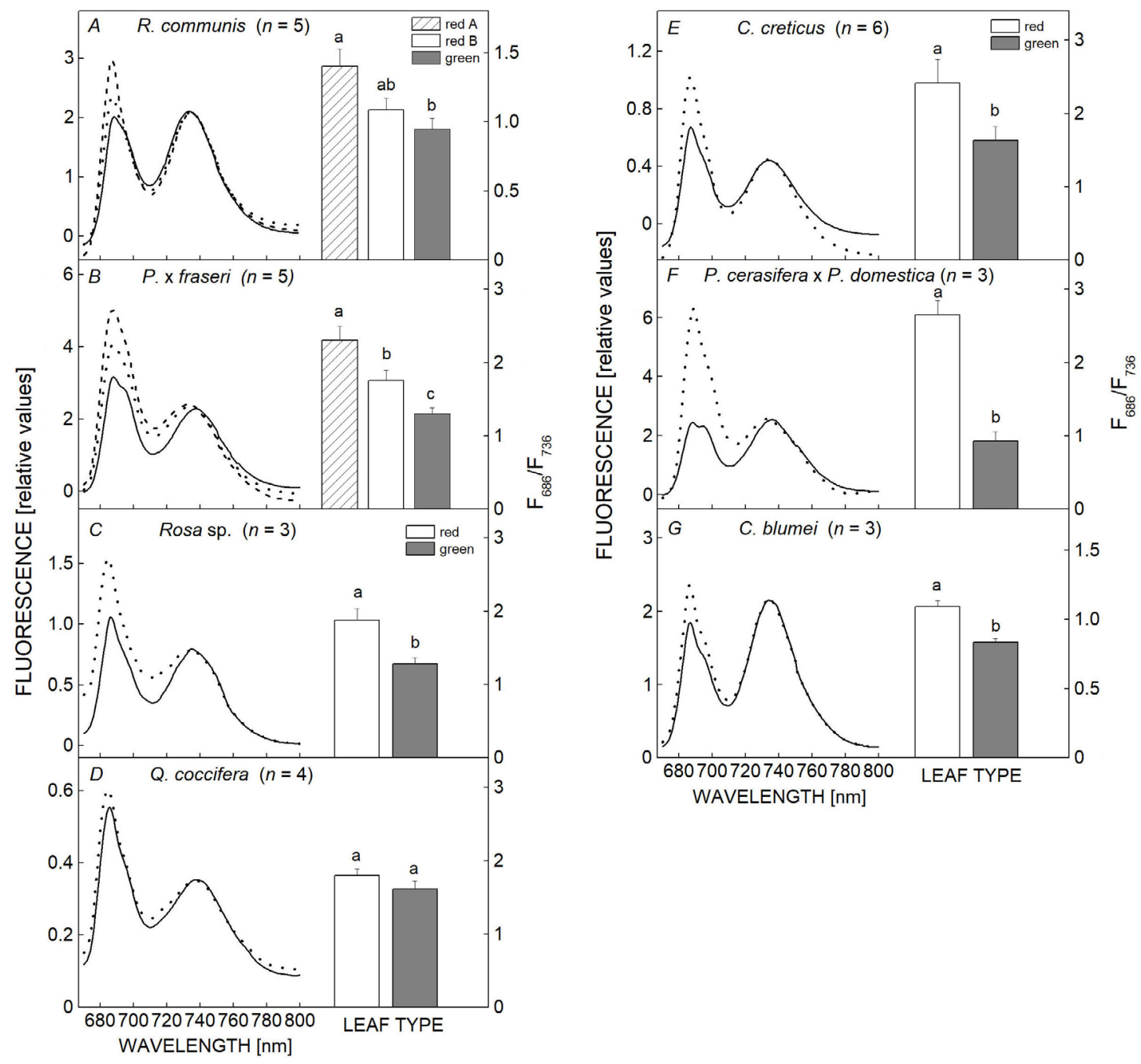

Fig. 1. $77 \mathrm{~K}$ fluorescence emission spectra recorded from isolated thylakoids of red (dotted line) and green (solid line) leaves of the indicated species. Fluorescence values of red leaves were normalized to those of the corresponding green ones at $\lambda=736 \mathrm{~nm}$. Bars represent means $\pm \mathrm{SE}$ of the $\mathrm{F}_{686} / \mathrm{F}_{736}$ ratio from red (white bar) and green (grey bar) leaves, calculated from the corresponding emission spectra. Different letters above bars indicate statistically significant differences $(p<0.05)$ between red and green leaves within each species. In Ricinus communis and Photinia $\times$ fraseri, two age classes of young leaves, red A (dashed line, hatched bar) and red B (dotted line, white bar) respectively, were used. In the case of Cistus creticus, the emission spectra and the corresponding $\mathrm{F}_{686} / \mathrm{F}_{736}$ ratios concern the 'red measuring period'. $n$ - number of independent preparations.

note that in the case of $C$. creticus, the above differences were observed only during the 'red' period of the year and were almost abolished when both phenotypes were green.

In $C$. creticus, anthocyanin concentration and the $\mathrm{F}_{686} / \mathrm{F}_{736}$ ratio was monitored for a period of six months, encompassing both the 'green' and the 'red' period of the year (Fig. 3). Measurements started before the commencement of anthocyanin accumulation in the red phenotype (first week of December), continued during the whole 'red period', and ended in late May, when both phenotypes were green again. As it is shown, there is no difference in the $\mathrm{F}_{686} / \mathrm{F}_{736}$ ratio (Fig. $3 B$ ) at the green period of the year (early to mid-December and May), when anthocyanin content was almost zero in both leaf types. Leaf redness appeared in the red phenotype in late December and anthocyanin concentration increased steeply within few days and more gradually thereafter (Fig. 3A). Anthocyanin content was maintained at high levels until mid-March, while in green leaves, it remained constantly low. During the same period a concurrent rise of the $\mathrm{F}_{686} / \mathrm{F}_{736}$ ratio was observed in both phenotypes, which, however, was more pronounced in red leaves. In particular, the $\mathrm{F}_{686} / \mathrm{F}_{736}$ ratio was gradually doubled in green leaves until mid-February and declined thereafter to reach the initial value in early April. In red leaves, the $\mathrm{F}_{686} / \mathrm{F}_{736}$ ratio increased more sharply, i.e., 3.3-fold in late January, and remained high until mid-March, as long as anthocyanin concentrations were at maximum. The values obtained in late-May correspond to new leaves (both green). 

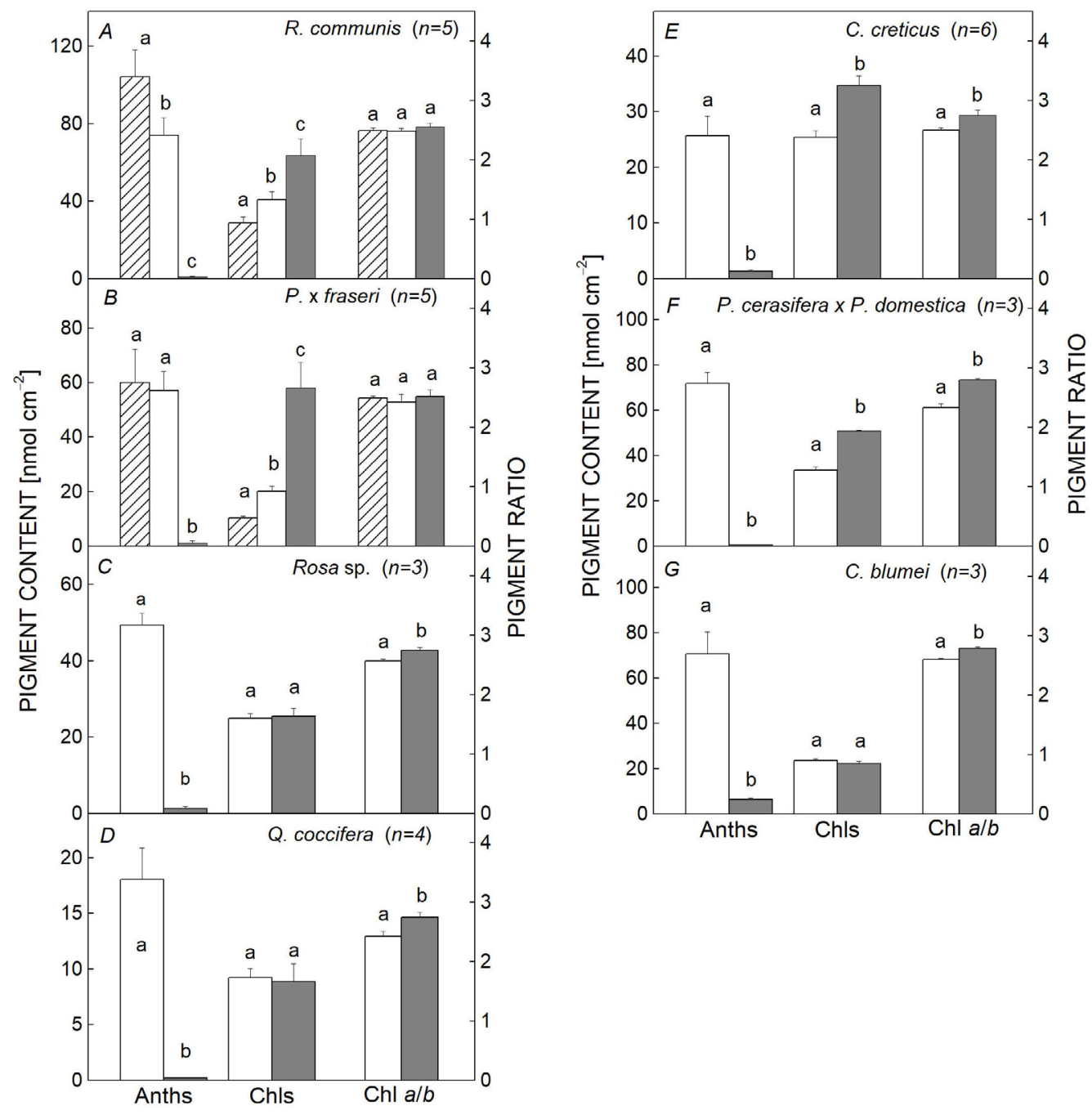

Fig. 2. Area-based total chlorophyll and anthocyanin concentrations and $\mathrm{Chl} a / b$ ratio from red (white bar) and green (grey bar) leaves or areas of the same species. In Ricinus communis and Photinia $\times$ fraseri, young leaves are presented by hatched bar (red A) and white bar (red B), respectively. Data are means \pm SE of the indicated $(n)$ independent extractions. Within each species, different letters indicate statistically significant differences $(p<0.05)$ in the measured parameters between red and green leaves. In Cistus creticus, values refer to the 'red measuring period'.

\section{Discussion}

To test whether the presence of an anthocyanin screen leads to a readjustment of photosystem stoichiometry, compatible to the shade acclimation hypothesis, we took advantage of the intra-individual, intra-species, or intraleaf variation in the expression of the anthocyanic trait of the selected plants (see the text table in 'Materials and methods'). In the first four species, transient leaf redness is developmentally determined (young red leaves which become green upon maturation), while in C. creticus is induced in mature leaves of certain individuals (red phenotype) by the combination of low temperatures and high light intensity during winter. The last two species display permanent anthocyanin accumulation either homogeneously in the whole leaf area (P. cerasifera) or in patchiness (C. blumei).
In $P . \times$ fraseri and $R$. communis, the compared leaves (young red $v s$. mature green) co-occur at the same individual, giving the opportunity to bypass possible species- and/or site-dependent effects in our measurements. Yet, unavoidably, the interference of the different developmental stage could not be excluded. To overcome possible age effects, in the next four species, leaves of the same physiological age (either young red $v s$. young green or mature red vs. mature green) were compared. Leaves were harvested from neighboring different individuals/ phenotypes of the same species growing under apparently similar environmental conditions to eliminate site effects of water and nutrient availability. Although speciesdependent differences could not be excluded in the case of $P$. cerasifera $\times P$. domestica, the compared mature green and red leaves co-occur on the same chimeric tree, possessing a permanently red ( $P$. cerasifera) and a 


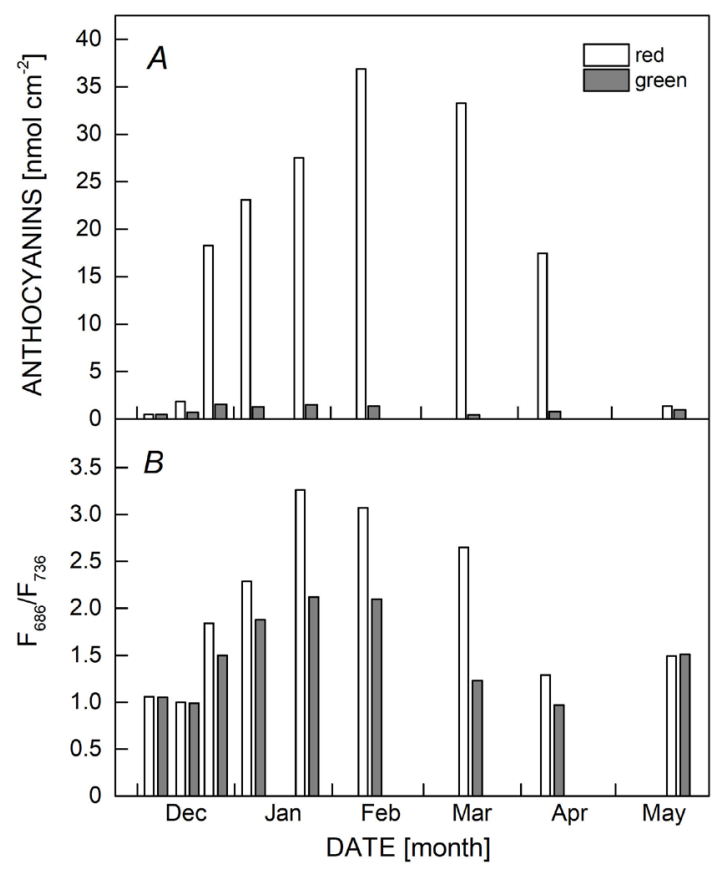

Fig. 3. Monitoring of anthocyanin contents $(A) \operatorname{andF_{686}} / \mathrm{F}_{736} \operatorname{ratio}(B)$ in mature leaves from green (grey bars) and red (open bars) phenotypes of Cistus creticus sampled on the indicated dates. On each measuring date, data are means from two independent extractions. Anthocyanin contents in green leaves were stable throughout the experimental period (average during red period: $1.32 \pm 0.26)$. Leaves senesce and fall from mid-April to mid-May in parallel with new leaf emergence. The May sampling concerns new green leaves for both phenotypes. Differences between the two phenotypes are significant only during the 'red' period.

permanently green $(P$. domestica) stem. Hence, both leaf types experience the same environmental limitations. Finally, C. blumei seems to fulfil all the requisites, since anthocyanin-rich and anthocyanin-less chlorophyllous regions from the same leaf were compared.

It is evident from our results that, compared to green, red leaves display higher emission intensity at $686 \mathrm{~nm}$ and, accordingly, higher $\mathrm{F}_{686} / \mathrm{F}_{736}$ values, indicative of an enhanced PSII/PSI ratio (Fig. 1). In all cases, the $77 \mathrm{~K}$ fluorescence spectra of isolated thylakoids showed a typical profile with two main peaks at 686 and $736 \mathrm{~nm}$, corresponding to the emissions of PSII core complex and LHCI-PSI, respectively. A shoulder at $695 \mathrm{~nm}$ was also observed, ascribable to CP47 emission. Since the peak at $686 \mathrm{~nm}$ was better defined in our normalized spectra, we used the fluorescence amplitude at this wavelength to estimate the PSII/PSI ratio (Andrizhiyevskaya et al. 2005, Tang et al. 2005, Velitchkova and Popova 2005, Lamb et al. 2018).

The significantly higher PSII/PSI ratio of red leaves was observed in all tested species (except of Q. coccifera, where the same was observed as a trend), regardless of the inductive agent of anthocyanin accumulation (ontogenetic or environmental) or their histological localization (epidermis or mesophyll). The latter agrees with previous findings suggesting that the absorption capacity of anthocyanins for green light is largely independent of their histological localization (Gould et al. 2002a, 2018), although in a comparative study with red and green leaves of Quintinia serrata, it was proposed that the tissue location of anthocyanins is important for the absorption profile of the leaf (Gould et al. 2002b). Moreover, as it was shown in C. creticus, where the differences between the two phenotypes were monitored during both the 'red' and the 'green' period, the increase of PSII/PSI ratio followed the pattern of anthocyanin accumulation (Fig. 3). The PSII/PSI ratio increased in both phenotypes during winter, indicating that photosystem stoichiometry was affected by several environmental factors, apart from incident light, such as low temperature (Walters 2005). Yet, the increase was more pronounced in red leaves and the differences were abolished when the red phenotype reverted to green. Accordingly, we may consider the above-mentioned increase as a response to the unbalanced light quality penetrating into a red leaf to regulate the excitation energy afforded in the two photosystems, analogous to the corresponding shade acclimation (Melis 1984, Chow et al. 1990, Anderson et al. 1995).

The light prevailing under natural shade (e.g., under a dense plant or forest canopy), apart from being of significantly lower intensity, it is relatively enriched in green and far-red while depleted in blue/red photons (Anderson et al. 1995, Lichtenthaler et al. 2013). Although, the light available under natural shade conditions tends to induce opposite effects in PSII/PSI ratio, i.e., a decrease due to low intensity and an increase due to uneven spectral distribution, the resulting adjustment of photosystem stoichiometry is considered rather an acclimation to spectral quality than intensity (Melis 1991, Anderson et al. 1995, Murchie and Horton 1998). When FR-enriched light reaches chloroplasts, PSI receives much more excitation energy compared to PSII, thus PSI operation is favored. Conversely, incident light enriched in blue/red photons preferentially excites PSII while PSI excitation is limiting for photosynthesis. As it has been repeatedly shown with plants grown under illumination of different quality, the enrichment of light in FR photons (i.e., PSI light) leads to an increase in the relative PSII/PSI ratio, to optimize light utilization by the two photosystems (Melis 1984, Glick et al. 1985, Chow et al. 1990, Melis 1991, Walters 2005). Accordingly, in true shade leaves, the PSII/PSI ratio is increased. The shade imposed by anthocyanin accumulation in a red leaf, however, apart from similarities to that of natural (i.e., canopy-like) conditions, displays also characteristic differences. Foliar anthocyanins, being almost always red (Harborne 1976), absorb strongly in the green/yellow and less in the blue wavebands, while they are transparent in far-red band. Concerning their absorbance ability in the red portion of the spectrum, it is considered negligible or at least very low (Karabourniotis et al. 1999, Gould et al. 2002b, Steyn et al. 2002, Kyparissis et al. 2007). As a result, while natural shade is green- and FR-enriched, the mesophyll of a red leaf is exposed to light severely depleted in the green/yellow portion of the visible spectrum. In addition, anthocyanins 
may also display an appreciable tail over the red band $(600-630 \mathrm{~nm})$, especially when they are conjugated with other (colorless) flavonoids, reducing even more the R/FR ratio compared to natural shade (Gould et al. 2018, Landi et al. 2021). Green and yellow light is better absorbed by PSII, whereas FR light is preferentially absorbed by PSI (Glick et al. 1985, Pfannschmidt 2005).

Several reports in the literature support the assumption that anthocyanin accumulation induces some morphological and physiological adjustments in red leaves, resembling, at least partly, with those displayed by the true shade (green) ones. Thus, compared to their green counterparts, red leaves tend to be thinner with lower LMA values, reduced $\mathrm{Chl} a / b$ and $\mathrm{Car} / \mathrm{Chls}$ ratios, lower net photosynthetic rate, and lower or similar area based total Chls (Burger and Edwards 1996, Manetas et al. 2003, Hughes and Smith 2007, Hughes et al. 2007, Kyparissis et al. 2007, Kytridis et al. 2008, Zeliou et al. 2009). In many cases, however, some traits of red leaves are not compatible to the shade-acclimation syndrome. According to Kyparissis et al. (2007), these deviations could be explained by the selective absorption of anthocyanins, especially in the green/yellow part of the spectrum, leading to thinner rather than shade leaves, in which the missing layers had the more shade characteristics.

As already mentioned in 'Introduction', a typical 'shade' character of leaves growing under a dense canopy is the lower Chl $a / b$ ratio, which reflects a higher investment in light harvesting (containing both $\mathrm{Chl} a$ and $b$ ) relative to core complexes (containing only $\mathrm{Chl} a$ ) and/or a higher PSII/PSI ratio (Anderson 1986, Murchie and Horton 1998, Lichtenthaler and Babani 2004, Hughes et al. 2007, Lichtenthaler et al. 2007). In a comparative study with anthocyanic and non-anthocyanic species (Hughes et al. 2007), Chl $a / b$ ratio increased gradually with leaf age in the developing red leaves (in parallel to the decline of anthocyanin content), while in the corresponding green ones, it remained relatively constant at much higher values during development. Accordingly, the reduced $\mathrm{Chl} a / b$ ratio in reds was attributed to the shade imposed by anthocyanins (Hughes et al. 2007). In the present study, red leaves/areas in five of our tested species displayed a lower Chl $a / b$ ratio compared to their green counterparts (Fig. 2C-G), regardless of their developmental stage (young or mature). Similarly, the lower Chl $a / b$ ratio in reds, ascribed to the shade imposed by anthocyanins, was reported in several previous investigations (Manetas et al. 2003, Hughes and Smith 2007, Hughes et al. 2007, Kyparissis et al. 2007, Zeliou et al. 2009). In $R$. communis and $P . \times$ fraseri, however, no differences were observed between the leaf types, despite the much higher anthocyanin content of red leaves (Fig. 2A,B). A possible explanation would be that chloroplasts in the mature leaves are self-shaded by the much higher $\mathrm{Chl}$ concentration (combined with the increased thickness), leading to similar $\mathrm{Chl} a / b$ values in green and red leaves. As it has been shown, changes of the chlorophyll content affect the proportion of 'shaded' chloroplasts within a leaf, which results in changes of the $\mathrm{Chl} a / b$ ratio (Walters 2005). Yet, in $P$. cerasifera $\times P$. domestica and C. creticus, where Chl concentration was also higher in green leaves, the above argument is not confirmed, unless if the steeper gradient of $\mathrm{Chl}$ content between red and green leaves of $R$. communis and $P . \times$ fraseri contributes more effectively to the modulation of $\mathrm{Chl} a / b$ ratio. In any case, in all tested species of the present study, red leaves displayed a higher PSII/PSI ratio, irrespective of the anthocyanin accumulation pattern and the difference in $\mathrm{Chl} a / b$ ratio. According to Murchie and Horton (1998), a change in Chl $a / b$ is not necessarily associated to changes in PSII/ PSI ratio.

\section{References}

Anderson J.M.: Photoregulation of the composition, function, and structure of thylakoid membranes. - Annu. Rev. Plant Phys. 37: 93-136, 1986.

Anderson J.M., Chow W.S., Park Y.-I.: The grand design of photosynthesis: Acclimation of the photosynthetic apparatus to environmental cues. - Photosynth. Res. 46: 129-139, 1995.

Andrizhiyevskaya E.G., Chojnicka A., Bautista J.A. et al.: Origin of the F685 and F695 fluorescence in Photosystem II. Photosynth. Res. 84: 173-180, 2005.

Archetti M., Döring T.F., Hagen S.B. et al.: Unravelling the evolution of autumn colours: an interdisciplinary approach. Trends Ecol. Evol. 24: 166-173, 2009.

Burger J., Edwards G.E.: Photosynthetic efficiency and photodamage by UV and visible radiation in red versus green leaf coleus varieties. - Plant Cell Physiol. 37: 395-399, 1996.

Chalker-Scott L.: Environmental significance of anthocyanins in plant stress responses. - Photochem. Photobiol. 70: 1-9, 1999.

Chow W.S., Melis A., Anderson J.M.: Adjustments of photosystem stoichiometry in chloroplasts improve the quantum efficiency of photosynthesis. - P. Natl. Acad. Sci. USA 87: 7502-7506, 1990.

Close D.C., Beadle C.L.: The ecophysiology of foliar anthocyanins. - Bot. Rev. 69: 149-161, 2003.

Glick R.E., McCauley S.W., Melis A.: Effect of light quality on chloroplast-membrane organization and function in pea. Planta 164: 487-494, 1985.

Gould K.S.: Nature's Swiss army knife: the diverse protective roles of anthocyanins in leaves. - J. Biomed. Biotechnol. 2004: 314-320, 2004

Gould K.S., Jay-Allemand C., Logan B.A. et al.: When are foliar anthocyanins useful to plants? Re-evaluation of the photoprotection hypothesis using Arabidopsis thaliana mutants that differ in anthocyanin accumulation. - Environ. Exp. Bot. 154: 11-22, 2018.

Gould K.S., Neill S., Vogelmann T.C.: A unified explanation for anthocyanins in leaves? - Adv. Bot. Res. 37: 167-192, 2002a.

Gould K.S., Vogelmann T.C., Han T., Clearwater M.J.: Profiles of photosynthesis within red and green leaves of Quintinia serrata A. Cunn. - Physiol. Plantarum 116: 127-133, 2002 b.

Harborne J.B.: The anthocyanin pigments. - In: Harborne J.B. (ed.): Comparative Biochemistry of the Flavonoids. Pp. 1-36. Academic Press, London 1976.

Hoch W.A., Zeldin E.L., McCowan B.H.: Physiological significance of anthocyanins during autumnal leaf senescence. Tree Physiol. 21: 1-8, 2001.

Hogewoning S.W., Wientjes E., Douwstra P. et al.: Photosynthetic quantum yield dynamics: from photosystems to leaves. Plant Cell 24: 1921-1935, 2012.

Hrazdina G., Wagner G.J., Siegelman H.W.: Subcellular localization of enzymes of anthocyanin biosynthesis in protoplasts. - Phytochemistry 17: 53-56, 1978. 
Hughes N.M.: Winter leaf reddening in 'evergreen' species. New Phytol. 190: 573-581, 2011.

Hughes N.M., Morley C.B., Smith W.K.: Coordination of anthocyanin decline and photosynthetic maturation in juvenile leaves of three deciduous tree species. - New Phytol. 175: 675-685, 2007.

Hughes N.M., Smith W.K.: Attenuation of incident light in Galax urceolata (Diapensiaceae): concerted influence of adaxial and abaxial anthocyanic layers on photoprotection. - Am. J. Bot. 94: 784-790, 2007.

Karabourniotis G., Bornman J.F., Liakoura V.: Different leaf surface characteristics of three grape cultivars affect leaf optical properties as measured with fibre optics: possible implication in stress tolerance. - Aust. J. Plant Physiol. 26: 47-53, 1999.

Krause G.H., Weis E.: Chlorophyll fluorescence and photosynthesis: the basics. - Annu. Rev. Plant Phys.42: 31349, 1991

Kyparissis A., Grammatikopoulos G., Manetas Y.: Leaf morphological and physiological adjustments to the spectrally selective shade imposed by anthocyanins in Prunus cerasifera. - Tree Physiol. 27: 849-857, 2007.

Kytridis V.-P., Karageorgou P., Levizou E., Manetas Y.: Intraspecies variation in transient accumulation of leaf anthocyanins in Cistus creticus during winter: evidence that anthocyanins may compensate for an inherent photosynthetic and photoprotective inferiority of the red-leaf phenotype. J. Plant Physiol. 165: 952-959, 2008.

Kytridis V.-P., Manetas Y.: Mesophyll versus epidermal anthocyanins as potential in vivo antioxidants: evidence linking the putative antioxidant role to the proximity of oxy-radical source. - J. Exp. Bot. 57: 2203-2210, 2006.

Lamb J.J., Røkke G., Hohmann-Marriott M.F.: Chlorophyll fluorescence emission spectroscopy of oxygenic organisms at 77 K. - Photosynthetica 56: 105-124, 2018.

Landi M., Agati G., Fini A. et al.: Unveiling the shade nature of cyanic leaves: A view from the "blue absorbing side" of anthocyanins. - Plant Cell Environ. 44: 1119-1129, 2021.

Lee D.W., Collins T.M.: Phylogenetic and ontogenetic influences on the distribution of anthocyanins and betacyanins in leaves of tropical plants. - Int. J. Plant Sci. 162: 1141-1153, 2001

Lev-Yadun S., Dafni A., Flaishman M.A. et al.: Plant coloration undermines herbivorous insect camouflage. - BioEssays 26: 1126-1130, 2004.

Lichtenthaler H.K., Ač A., Marek M.V. et al:: Differences in pigment composition, photosynthetic rates and chlorophyll fluorescence images of sun and shade leaves of four tree species. - Plant Physiol. Bioch. 45: 577-588, 2007.

Lichtenthaler H.K., Babani F., Navrátil M., Buschmann C.: Chlorophyll fluorescence kinetics, photosynthetic activity, and pigment composition of blue-shade and half-shade leaves as compared to sun and shade leaves of different trees. Photosynth. Res. 117: 355-366, 2013.

Lichtenthaler H.K., Babani F.: Light adaptation and senescence of the photosynthetic apparatus: changes in pigment composition, chlorophyll fluorescence parameters and photosynthetic activity during light adaptation and senescence of leaves. In: Papageorgiou G.C., Govindjee (ed.): Chlorophyll a Fluorescence: A Signature of Photosynthesis. Advances in Photosynthesis and Respiration. Pp. 713-736. Springer, Dordrecht 2004.

Lichtenthaler H.K., Wellburn A.R.: Determinations of total carotenoids and chlorophylls $a$ and $b$ of leaf extracts in different solvents. - Biochem. Soc. T. 11: 591-592, 1983.

Lindoo S.J., Caldwell M.M.: Ultraviolet-B radiation-induced inhibition of leaf expansion and promotion of anthocyanin production - lack of involvement of low irradiance phytochrome system. - Plant Physiol. 61: 278-282, 1978.

Manetas Y.: Why some leaves are anthocyanic and why most anthocyanic leaves are red? - Flora 201: 163-177, 2006.

Manetas Y., Petropoulou Y., Psaras G.K., Drinia A.: Exposed red (anthocyanic) leaves of Quercus coccifera display shade characteristics. - Funct. Plant Biol. 30: 265-270, 2003.

Melis A.: Light regulation of photosynthetic membrane structure, organization and function. - J. Cell. Biochem. 24: 271-285, 1984.

Melis A.: Dynamics of photosynthetic membrane composition and function. - BBA-Bioenergetics 1058: 87-106, 1991.

Merzlyak M.N., Chivkunova O.B., Solovchenko A.E., Naqvi K.R.: Light absorption by anthocyanins in juvenile, stressed, and senescing leaves. - J. Exp. Bot. 59: 3903-3911, 2008.

Murchie E.H., Horton P.: Contrasting patterns of photosynthetic acclimation to the light environment are dependent on the differential expression of the responses to altered irradiance and spectral quality. - Plant Cell Environ. 21: 139-148, 1998.

Murray J.R., Hackett W.P.: Dihydroflavonol reductase activity in relation to differential anthocyanin accumulation in juvenile and mature phase Hedera helix L. - Plant Physiol. 97: 343$351,1991$.

Neill S., Gould K.S.: Optical properties of leaves in relation to anthocyanin concentration and distribution. - Can. J. Bot. 77: 1777-1782, 2000.

Papageorgiou G.C., Govindjee: Chlorophyll a Fluorescence: A Signature of Photosynthesis. Advances in Photosynthesis and Respiration. Pp. 818. Springer, Dordrecht 2004.

Pfannschmidt T.: Acclimation to varying light qualities: toward the functional relationship of state transitions and adjustment of photosystem stoichiometry. - J. Phycol. 41: 723-725, 2005.

Pietrini F., Massacci A.: Leaf anthocyanin content changes in Zea mays L. grown at low temperature: significance for the relationship between the quantum yield of PSII and the apparent quantum yield of $\mathrm{CO}_{2}$ assimilation. - Photosynth. Res. 58: 213-219, 1998.

Šiffel P., Hunalová I., Roháček K.: Light-induced quenching of chlorophyll fluorescence at $77 \mathrm{~K}$ in leaves, chloroplasts and Photosystem II particles. - Photosynth. Res. 65: 219-229, 2000.

Steyn W.J., Wand S.J.E., Holcroft D.M., Jacobs G.: Anthocyanins in vegetative tissues: a proposed unified function in photoprotection. - New Phytol. 155: 349-361, 2002.

Tang Y., Wen X., Lu C.: Differential changes in degradation of chlorophyll-protein complexes of photosystem I and photosystem II during flag leaf senescence of rice. - Plant Physiol. Bioch. 43: 193-201, 2005.

Velitchkova M., Popova A.: High light-induced changes of $77 \mathrm{~K}$ fluorescence emission of pea thylakoid membranes with altered membrane fluidity. - Bioelectrochemistry 67: 81-90, 2005.

Walters R.G.: Towards an understanding of photosynthetic acclimation. - J. Exp. Bot. 56: 435-447, 2005.

Weis E.: Chlorophyll fluorescence at $77 \mathrm{~K}$ in intact leaves: characterization of a technique to eliminate artifacts related to self-absorption. - Photosynth. Res. 6: 73-86, 1985.

Yiotis C., Petropoulou Y., Manetas Y.: Evidence for lightindependent and steeply decreasing PSII efficiency along twig depth in four tree species. - Photosynthetica 47: 223231, 2009.

Zeliou K., Manetas Y., Petropoulou Y.: Transient winter leaf reddening in Cistus creticus characterizes weak (stresssensitive) individuals, yet anthocyanins cannot alleviate the adverse effects on photosynthesis. - J. Exp. Bot. 60: 3031$3042,2009$.

(C) The authors. This is an open access article distributed under the terms of the Creative Commons BY-NC-ND Licence. 